\title{
Mitigating wind noise with a spherical microphone array
}

\author{
Sipei Zhao ${ }^{\text {a) }}$ and Matthew Dabin \\ School and Engineering, RMIT University, Melbourne, Australia
}

Eva Cheng, Xiaojun Qiu, and lan Burnett

Centre for Audio, Acoustics and Vibration, Faculty of Engineering and Information Technology, University of Technology Sydney, Sydney, Australia

Jacob Chia-chun Liu

Department of Water Resources and Environmental Engineering, Tamkang University, New Taipei City, Taiwan

(Received 25 February 2018; revised 7 October 2018; accepted 19 November 2018; published online 10 December 2018)

\begin{abstract}
This paper utilizes a rigid spherical microphone array to reduce wind noise. In the experiments conducted, a loudspeaker is used to reproduce the desired sound signal and an axial fan is employed to generate wind noise in an anechoic chamber. The sound signal and wind noise are measured separately with the spherical microphone array and analyzed in the spherical harmonic domain. The wind noise is found to be irregularly distributed in the spherical harmonic domain, distinct from the sound signal which is concentrated in the first few spherical harmonic modes. This difference is utilized to reduce wind noise without degrading the desired sound pressure level (SPL) by use of a low pass filter method in the spherical harmonic domain. Experimental results with both singletonal and multi-tonal sound signals demonstrate that the proposed method can reduce wind noise by more than $10 \mathrm{~dB}$ in the frequency range below $500 \mathrm{~Hz}$. The SPL of the desired sound signal can be extracted from wind noise with an error within $1.0 \mathrm{~dB}$, even when the sound level is $8 \mathrm{~dB}$ lower than wind noise. (C) 2018 Acoustical Society of America. https://doi.org/10.1121/1.5082290
\end{abstract}

$[\mathrm{DKW}]$

Pages: $3211-3220$

\section{INTRODUCTION}

Acoustic measurements in outdoor environments and ventilation ducts are often contaminated by wind noise due to the turbulent pressure fluctuations (Raspet et al., 2006; Shepherd and La Fontaine, 1986). Various methods have been explored in the past to attenuate wind noise, which can be categorized into methods that utilize physical structures and signal processing methods (Shust, 1998).

Porous microphone windscreens are the most popular structures for wind noise reduction (WNR), especially in outdoor acoustic measurements (van den Berg, 2006). Strasberg (1988) analyzed the wind noise inside spherical and cylindrical porous windscreens with dimensional analysis and found that the wind noise spectra measured by different researchers form a single curve when plotted against the Strouhal number. Morgan and Raspet (1992) compared the noise reduction characteristics of various spherical open-cell foam windscreens and showed that the spherical windscreens have better performance than the streamlined windscreens. Zhao et al. (2017b) measured the WNR of five $90 \mathrm{~mm}$ diameter porous microphone windscreens with porosity from 20 to 60 pores per inch (PPI) and showed that the 40 PPI windscreen exhibited the best performance.

Nonporous windscreens are also used to reduce wind noise, especially in the infrasonic range. Collier et al. (2014) investigated the WNR performance of three semi-porous fabric domes composed of different materials and showed

\footnotetext{
a)Electronic mail: sipei.zhao@uts.edu.au
}

that the primary source of low frequency wind noise was due to the pressure fluctuations on the surface of the domes. Shams et al. (2005) developed compact nonporous microphone windscreens for infrasonic acoustic measurements based on the assumption that the infrasound can penetrate any barrier of practical thickness while the wind fluctuations are blocked by the solid nonporous walls. The experimental results showed that a windscreen composed of closed-cell polyurethane foam with an internal diameter and height of $3 \times 9$ in. $^{2}$, and a wall thickness of $0.5 \mathrm{in}$. achieved the best performance. Dauchez et al. (2016) studied the performance of a windscreen constructed from a squared plate coupled with a nonporous cavity and showed the mechanism of WNR to be the spatial averaging of the pressure fluctuations over the plate.

Hedlin and Raspet (2003) measured the WNR of a cylindrical barrier of $2 \mathrm{~m}$ height with a diameter of $5.5 \mathrm{~m}$, and compared to that of the rosette filters with diameters of 18 and $70 \mathrm{~m}$ in atmosphere. It was found that the rosette filters only produce reductions if the turbulence scale is smaller than the diameter of the rosette, and the cylindrical barrier has large reductions only when the scale size of the turbulence is smaller than the height of the barrier. Abbott et al. (2015) optimized the WNR of a porous wind fence enclosure which is $2.9 \mathrm{~m}$ high and has a diameter of $5.0 \mathrm{~m}$, and found that the best reduction was achieved with a surface porosity between $40 \%$ and $55 \%$, supplemented by a secondary windscreen.

In addition to the above methods adopting physical structures to reduce wind noise, signal processing techniques have also been widely studied. Chung (1977) proposed a 
coherence function method for flow noise rejection using three pressure transducers based on the assumption that the flow noise is mutually uncorrelated at each transducer. However, this assumption is not valid in the lower frequency range which corresponds to the large scale turbulent eddies. Shields (2005) employed a three-axis orthogonal microphone array with 10 sensors in each arm to measure outdoor wind noise and showed that the time domain correlation as a function of sensor separation varies as $\mathrm{e}^{-3.2 X} \cos (2 \pi X)$ in the downwind direction and decays as $\mathrm{e}^{-7 Y}$ in the crosswind direction, where $X$ and $Y$ are the separation in wavelengths in the downwind and crosswind directions, respectively. Wilson et al. (2007) measured the outdoor wind noise with a $7 \times 7$ planar horizontal microphone array and found that the wind noise is substantially correlated for microphone separations smaller than the size of the turbulent eddies. Wilson and White (2010) analyzed the spatial and temporal characteristics of the wind noise, and discriminated the sound signal from wind noise with a Gaussian-mixture-model classifier.

In an alternative approach, McGuinn et al. (1997) proposed reducing the flow induced noise in ducts by use of the adaptive least mean square (LMS) algorithm to filter out the flow velocity fluctuations measured by a hot wire anemometer before subtraction from the pressure fluctuations measured by a microphone. This was based on the assumption that the flow velocity fluctuations are highly correlated with the flow induced noise. Similarly, Shust and Rogers (1998) studied the performance of wind noise removal from outdoor microphones using velocity measurements from a four channel anemometer and a simple model to transform the wind velocity fluctuations into wind noise estimates based on the Bernoulli equation. Unfortunately, the Bernoulli equation is only valid for fluid flows without turbulence and the coherence between the hot wire anemometer signal and the microphone signal is lower in outdoor environments, thus the performance was unsatisfactory (Shust, 1998).

In contrast to the abovementioned research, this paper utilizes a rigid spherical microphone array to mitigate wind noise. The sound signal and wind noise are first measured with the spherical microphone array separately and analyzed in the spherical harmonic domain. Then, a low pass filter method in the spherical harmonic domain is used to reduce the wind noise but retain the desired sound signal. Experimental results demonstrate the feasibility of the proposed method in situations where the wind noise spectrum masks the sound signal.

\section{METHOD}

A low pass filter method in the spherical harmonic domain (illustrated in Fig. 1) is proposed to reduce wind noise captured by a spherical microphone array. The recorded signals are first transformed to the spherical harmonic domain via the discrete spherical Fourier transform (Rafaely, 2015),

$$
\mathbf{p}_{n m}=\mathbf{Y}^{\dagger} \mathbf{p}
$$

\section{Input: recorded signal}

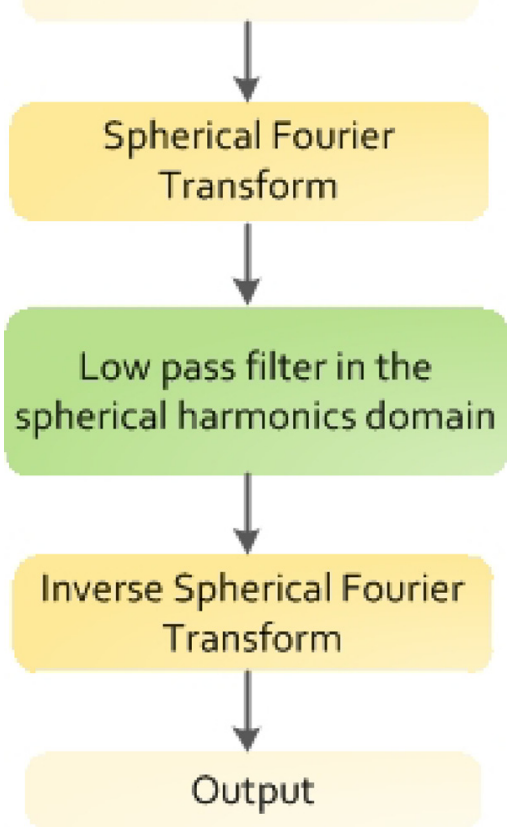

FIG. 1. (Color online) Diagram of the proposed method.

where the superscript ${ }^{\dagger}$ denotes the pseudo inverse operation, $\mathbf{p}=\left[p\left(f, \theta_{1}, \phi_{1}\right), \ldots, p\left(f, \theta_{q}, \phi_{q}\right), \ldots, p\left(f, \theta_{Q}, \phi_{Q}\right)\right]^{\mathrm{T}}, p\left(f, \theta_{q}\right.$, $\left.\phi_{q}\right)$ is the signal at the $q$ th microphone at frequency $f,\left(\theta_{q}\right.$, $\left.\phi_{q}\right)$ denotes the elevation angle $\left(\theta_{q}\right)$ and azimuth angle $\left(\phi_{q}\right)$ of the $q$ th microphone, and $Q=64$ is the number of microphones in the spherical microphone array. $\mathbf{p}_{n m}=\left[p_{00}(f)\right.$, $\left.p_{1(-1)}(f), p_{10}(f), p_{11}(f), \ldots, p_{N N}(f)\right]^{\mathrm{T}}$ are the spherical harmonic coefficients, $N$ is the highest order of the decomposition, and the matrix $\mathbf{Y}$ of dimensions $Q \times(N+1)^{2}$ is given by

$$
\mathbf{Y}=\left[\begin{array}{ccccc}
Y_{0}^{0}\left(\theta_{1}, \phi_{1}\right) & \cdots & Y_{n}^{m}\left(\theta_{1}, \phi_{1}\right) & \cdots & Y_{N}^{N}\left(\theta_{1}, \phi_{1}\right) \\
\vdots & \ddots & \vdots & \ddots & \vdots \\
Y_{0}^{0}\left(\theta_{q}, \phi_{q}\right) & \cdots & Y_{n}^{m}\left(\theta_{q}, \phi_{q}\right) & \cdots & Y_{N}^{N}\left(\theta_{q}, \phi_{q}\right) \\
\vdots & \ddots & \vdots & \ddots & \vdots \\
Y_{0}^{0}\left(\theta_{Q}, \phi_{Q}\right) & \cdots & Y_{n}^{m}\left(\theta_{Q}, \phi_{Q}\right) & \cdots & Y_{N}^{N}\left(\theta_{Q}, \phi_{Q}\right)
\end{array}\right]
$$

where $Y_{n}^{m}(\theta, \phi)$ is the spherical harmonic function of order $n$ $(n=0,1, \ldots, N)$ and degree $m(m=-n,-n+1, \ldots, 0,1, \ldots$, $n)$, which is defined as

$$
Y_{n}^{m}(\theta, \phi)=\sqrt{\frac{2 n+1}{4 \pi} \frac{(n-m) !}{(n+m) !}} P_{n}^{m}(\cos \theta) e^{i m \phi},
$$

where $P_{n}^{m}(\cdot)$ is the associated Legendre functions, $i$ is the imaginary unit, and $(\cdot)$ ! is the factorial operator.

Different modes of the spherical harmonic function represent different spatial patterns. For example, the first mode $Y_{0}^{0}(\theta, \phi)$ represents a monopole pattern, the spherical harmonic of the order $n=1$ are dipole patterns, and higher modes have more complex patterns (Rafaely, 2015). For a low frequency sound signal with long wavelength, the 
spherical microphone array has little effect on the sound propagation and most of the sound energy is focused in the first few modes of the spherical harmonic. For example, taking $\lambda>5 D$, the frequency $f$ should be less than $344 \mathrm{~Hz}$ ( $\lambda=c / f, c=344 \mathrm{~m} / \mathrm{s}$ is the speed of sound, $D=0.2 \mathrm{~m}$ ) for the spherical microphone array to have little effect on the sound propagation. However, for wind noise, the distribution of the noise energy along the sphere shows more complex patterns. This will be illustrated by the experimental results in Sec. III.

In consideration of the abovementioned difference between the sound signal and wind noise in the spherical harmonic domain, this paper proposes to mitigate wind noise by applying a low pass filter to the spherical harmonic coefficients, i.e.,

$$
\mathbf{p}_{n m}^{\prime}=\mathbf{w}_{n m} \cdot \mathbf{p}_{n m},
$$

where $\mathbf{p}_{n m}^{\prime}$ are the filtered spherical harmonic coefficients, $\mathbf{w}_{n m}=\left[w_{00}, w_{1(-1)}, w_{10}, w_{11}, \ldots, w_{N N}\right]^{\mathrm{T}}$ are the low pass filter coefficients. For brevity without loss of generality, a simple rectangular low pass filter in the spherical harmonic domain is used here, i.e.,

$$
w_{n m}= \begin{cases}1, & l \leq M, \\ 0, & l>M,\end{cases}
$$

where $l=n(n+1)+m+1$ is the mode number, and $M$ is the threshold mode number above which the spherical harmonics are filtered out. The low pass filtering in the spherical harmonic domain physically represents removing the higher order more complex patterns in the spatial domain, but retaining the monopole or the first few simple patterns.

After low pass filtering in the spherical harmonic domain, the spatial domain signals can be derived from the low pass filtered harmonic coefficients with the discrete inverse spherical Fourier transform

$$
\mathbf{p}^{\prime}=\mathbf{Y} \mathbf{p}_{n m}^{\prime},
$$

where $\mathbf{p}^{\prime}$ is the obtained sound signal after WNR. To quantify the WNR performance, WNR is defined as (Zhao et al., 2018)

$$
W N R(f)=10 \log _{10} \frac{P_{1}(f)}{P_{2}(f)} \quad(\mathrm{dB}),
$$

where $P_{1}(f)$ and $P_{2}(f)$ are the power spectral density of the wind noise before and after reduction.

It is noteworthy that the proposed method here is different from the beamforming methods. Beamforming methods are essentially spatial filters, which pick up sound signals from one direction and attenuate the signals from other directions. Therefore, for wind noise from the same direction as the sound signals, beamforming methods cannot reduce wind noise without degrading the sound signals. In contrast, the proposed method utilized the difference in spatial distribution between the sound signals and wind noise, which is independent of the sound signal direction. Even if the wind noise comes from the same direction as the sound signals, the wind noise can still be reduced with the proposed method.

The advantage of the proposed method is that the desired sound signal can be extracted from wind noise even when the sound signal is much lower than the wind noise. In addition, contrasting with existing WNR structures, e.g., large spatial filters (DeWolf et al., 2013) and wind fence enclosures (Abbott et al., 2015), the proposed method uses only a portable spherical microphone array, which is convenient for outdoor noise measurements. Finally, the proposed method is flexible and can be extended to spherical beamforming for future sound source localization. The feasibility of the proposed method will be verified by experiments and discussed in Sec. III.

\section{EXPERIMENTS AND DISCUSSIONS}

\section{A. Experiment setup}

The experiments were performed in an anechoic chamber with the experimental setup illustrated in Fig. 2. A commercial axial fan was used to generate wind noise and a B\&K type 4295 omnidirectional loudspeaker was employed to produce the sound signal. A Visisonics spherical microphone array with 64 microphones on a $20 \mathrm{~cm}$ diameter rigid sphere was utilized to measure the pressure fluctuations due to the sound signal and wind noise (VisiSonics, 2015). The frequency range of the Panasonic WM-61 A electret condenser microphone used in the Visisonics spherical array is $20 \mathrm{~Hz}-16 \mathrm{kHz}$. The spherical microphone array was placed in the middle of the anechoic chamber, and the fan and the

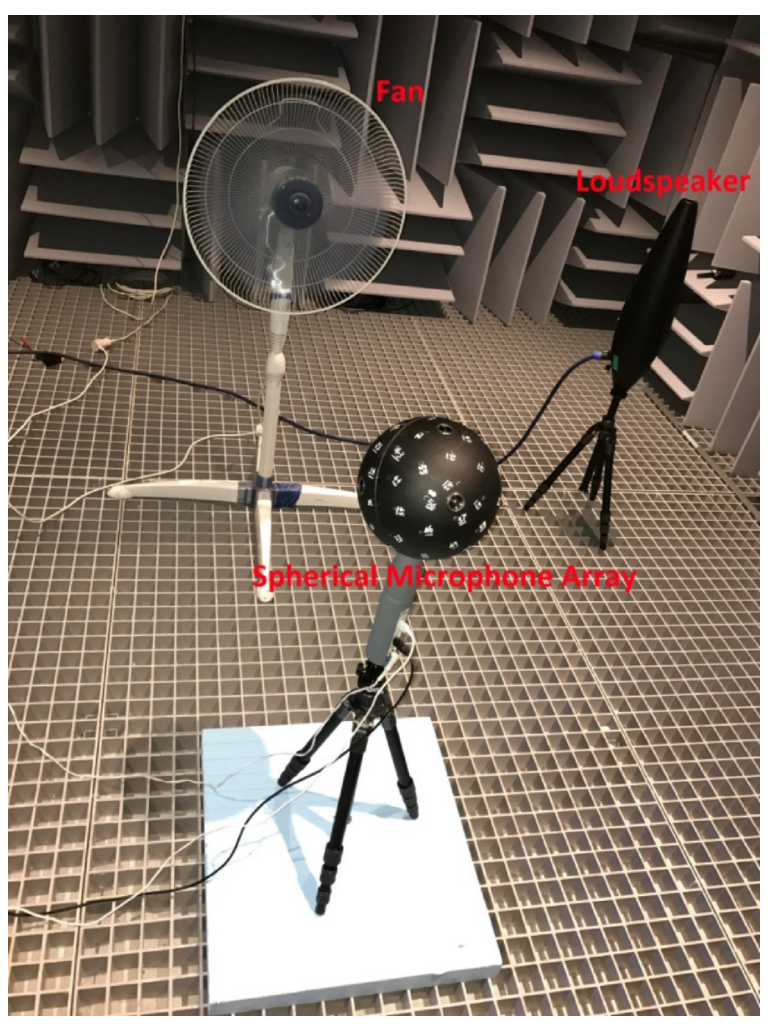

FIG. 2. (Color online) Experimental setup. 
loudspeaker were both $1.4 \mathrm{~m}$ away from the spherical microphone array. The fan was located at a direction of $\left(90^{\circ}, 0^{\circ}\right)$ relative to the spherical microphone array, where $(\theta, \phi)$ denotes the elevation angle and azimuth angle. The loudspeaker was placed at a variety of positions, but only results for the same direction as the fan $\left(90^{\circ}, 0^{\circ}\right)$ are presented here for brevity because the results for other directions are similar.

In the experiments, the sound signal was recorded first when the loudspeaker was active without the fan running, and then the wind noise was recorded when the fan was running alone. Finally, the sound signal, contaminated with wind noise, was recorded with both the loudspeaker and fan active. The characteristics of the sound signal and wind noise were analyzed in the spherical harmonic domain first, and then the difference between them was utilized to filter out wind noise in the spherical harmonic domain.

Figure 3 compares the recorded time domain signals at the front of the sphere near the stagnation point $\left(100^{\circ}, 0^{\circ}\right)$ and at the rear of the sphere $\left(100^{\circ}, 180^{\circ}\right)$. It can be observed from Fig. 3(a) that the low frequency sound signal at $150 \mathrm{~Hz}$ is similar at both the front and rear of the sphere, indicating that the sound signal is uniform around the sphere. The time axis in Fig. 3(a) is zoomed to be $0-0.1 \mathrm{~s}$ for the waveform details to be observed. In contrast, Fig. 3(b) shows that the wind noise at the front of the sphere is much higher than that at the rear, implying a complex distribution pattern of wind noise around the sphere. The correlation coefficients of the wind noise across each pair of microphones are found to be less than 0.3 , showing that the wind noises are uncorrelated around the sphere.

Figures 3(c) and 3(d) present the wind noise contaminated sound signal when the sound pressure level (SPL) of the sound signal is $10 \mathrm{~dB}$ higher and $3 \mathrm{~dB}$ lower than the wind noise, respectively, and clearly show that the sound signal is significantly distorted by the wind noise in the time domain. It is noteworthy that in Fig. 3(c), the sound signal is a tonal signal at $150 \mathrm{~Hz}$, which is higher than the wind noise at $150 \mathrm{~Hz}$. However, the wind noise is broadband signals, so pressure fluctuations of the wind noise in the time domain is larger than the tonal sound signal. While the spectral and spatial characteristics of the wind noise and sound signals are quite distinct, separation is difficult in the time domain. Hence, on the basis of the spherical microphone signals, the
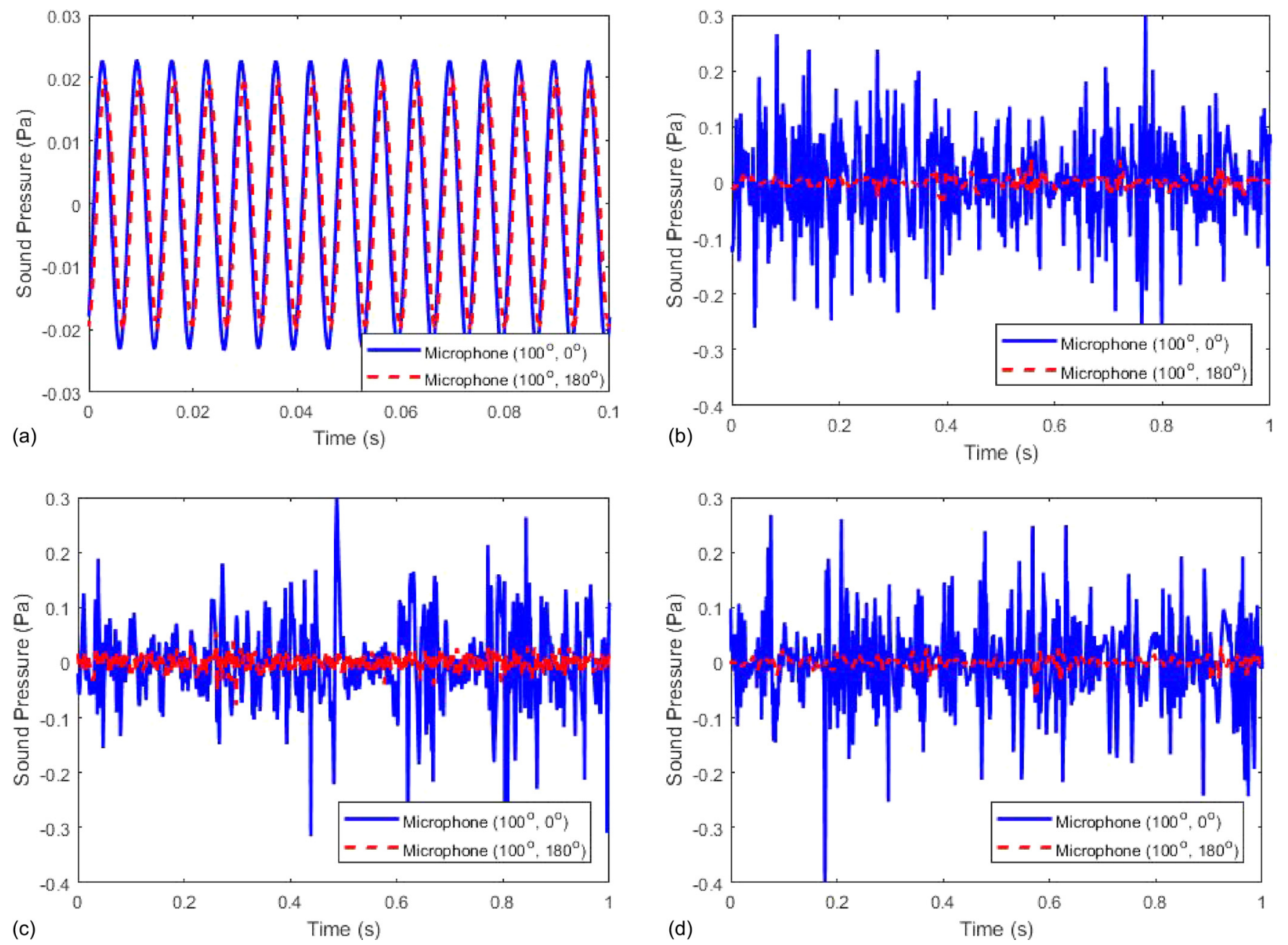

FIG. 3. (Color online) Comparison of the time domain signals measured at the front $\left(100^{\circ}, 0^{\circ}\right)$ and the rear $\left(100^{\circ}, 180^{\circ}\right)$ of the rigid spherical microphone array: (a) the $150 \mathrm{~Hz}$ tonal sound signal, (b) the wind noise, and the wind noise contaminated sound signal when the sound signal is (c) $10 \mathrm{~dB}$ higher, and (d) $3 \mathrm{~dB}$ lower than the wind noise. The mean wind speed at the spherical microphone array is $U=4.2 \mathrm{~m} / \mathrm{s}$. 
analysis was performed in the spherical harmonic domain in Sec. III B.

\section{B. Sound signal in the spherical harmonic domain}

The measured sound pressure are substituted into Eq. (1) to calculate the spherical harmonic coefficients $\mathbf{p}_{\mathrm{nm}}$, and the results for the sound signal without the fan running are shown in Fig. 4 at 150 and $500 \mathrm{~Hz}$, respectively. In the calculation, the sample length for spherical harmonic transform was $1 \mathrm{~s}$ with a sampling frequency of $44.1 \mathrm{kHz}$. The fast Fourier transform (FFT) length was 65 536, which is the next power of 2 of the sample length. For time dependent sound signals and wind noise, the proposed method can be applied every second. Therefore, a nearly real time noise monitoring system can be built based on the proposed method for long term measurements. The highest order $N=6$, so there are $L=(N+1)^{2}=49$ modes in the spherical harmonic decomposition. The abscissa mode number in Fig. 3 is $l=n(n+1)+m+1$, where $l=1$ represents the first mode $\mathrm{Y}_{0}^{0}(\theta, \phi)$ and the $l=49$ indicates the highest mode $\mathrm{Y}_{N}^{N}(\theta, \phi)$.

Figure 4(a) shows that the spherical harmonic coefficient for the first mode is much larger than that for higher modes at $150 \mathrm{~Hz}$, indicating that the sound energy is predominantly in the first mode. To quantify the percentage of sound energy contained in the first $L_{0}$ modes, the cumulative energy ratio is calculated as

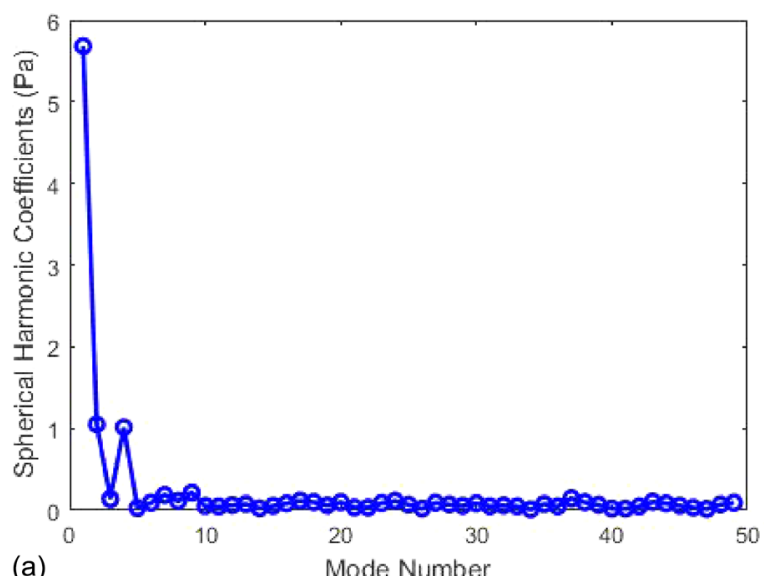

(a)

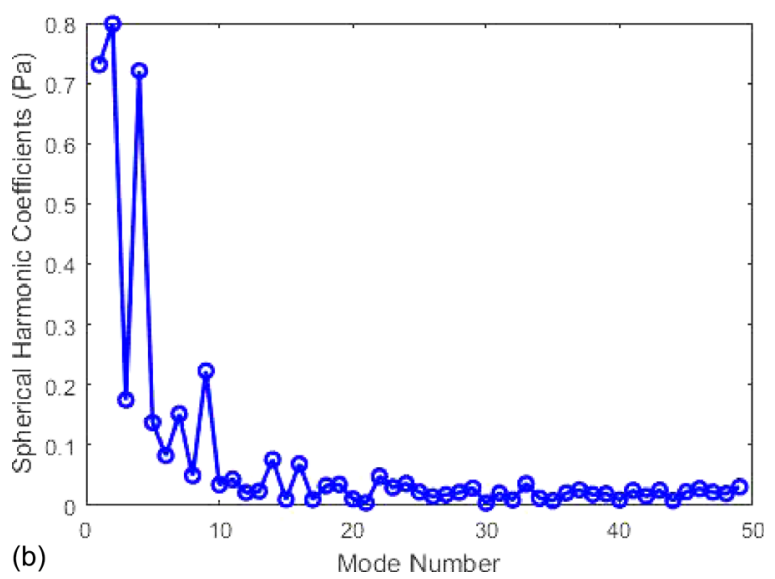

FIG. 4. (Color online) The spherical harmonic coefficients for the tonal sound signal at frequency of (a) $150 \mathrm{~Hz}$ and (b) $500 \mathrm{~Hz}$.
TABLE I. The cumulative energy ratio $(\%)$ for the sound signal.

\begin{tabular}{lcccccc}
\hline \hline $\operatorname{Modes}\left(L_{0}\right)$ & 1 & 2 & 3 & 4 & 5 & 10 \\
\hline $150 \mathrm{~Hz}$ & 0.930 & 0.961 & 0.962 & 0.992 & 0.992 & 0.995 \\
$500 \mathrm{~Hz}$ & 0.288 & 0.632 & 0.649 & 0.928 & 0.959 & 0.993 \\
\hline \hline
\end{tabular}

$$
R=\frac{\sum_{l=1}^{L_{0}} C_{l}^{2}}{\sum_{l=1}^{L} C_{l}^{2}} \times 100 \%
$$

where $C_{l}$ denotes the $l$ th spherical harmonic coefficient, $L$ is the total number of spherical harmonic modes. The accumulative energy ratio for the sound signal at 150 and $500 \mathrm{~Hz}$ corresponding to Fig. 4 is summarized in Table I. It is clear that $93.0 \%$ of the sound energy is contained in the first mode at $150 \mathrm{~Hz}$.

In the acoustic measurements, the error in the SPL is within $0.5 \mathrm{~dB}$ if $90 \%$ of the sound energy is captured. This implies that the measurement accuracy can be restricted to $0.5 \mathrm{~dB}$ by neglecting the higher modes but only retaining the first mode. To further verify this result, the low pass filter in the spherical harmonic domain with $M=1$ is used in Eq. (4) to calculate the filtered spherical harmonic coefficients, which are substituted in Eq. (6) to obtain the filtered signal. Comparison of the filtered signal with the original signal in Fig. 5(a) shows that the original SPL at $150 \mathrm{~Hz}$ is clearly extracted from the first mode with an error of $0.4 \mathrm{~dB}$.

Similarly, Fig. 4(b) shows that at $500 \mathrm{~Hz}$, the spherical harmonic coefficients in the first few modes are much larger than those in higher modes, and Table I shows that $92.8 \%$ sound energy is focused in the first four modes. A low pass filter in the spherical harmonic domain with $M=5$ and $M=1$ is applied and the filtered signal compared with the original signal in Fig. 5(b). It can be seen that the desired SPL at $500 \mathrm{~Hz}$ can be extracted from the first five modes with an error of $0.3 \mathrm{~dB}$. However, if only the first mode is utilized, the restored SPL is $5.5 \mathrm{~dB}$ lower than the original signal, which indicates that some of the sound energy is lost in the process.

The above results demonstrate that over $90 \%$ sound energy is contained in the first spherical harmonic mode at $150 \mathrm{~Hz}$ and in the first four modes at $500 \mathrm{~Hz}$. Hence, a low pass filter in the spherical harmonic domain can restore the original SPL with an error less than $0.5 \mathrm{~dB}$, and the threshold mode number $M$ needs to be tuned for different frequency sound signals. For higher frequency sound signals, higher modes are needed to restore the SPL, as shown in Table I. However, in the application scenario of WNR, the frequency range below $500 \mathrm{~Hz}$ is of greater interest because previous measurement results showed that wind noise above $500 \mathrm{~Hz}$ is below $40 \mathrm{~dB}$ in both indoor and outdoor environments (Zhao et al., 2016, 2017a).

\section{Wind noise in the spherical harmonic domain}

The wind noise was measured when the fan was running alone. In the experiment, the fan ran at three different 



FIG. 5. (Color online) Comparison of the original signal with the spherical harmonic domain filtered signal for the tonal sound at the frequency of (a) $150 \mathrm{~Hz}$ and (b) $500 \mathrm{~Hz}$.

speeds, and the mean wind speeds around the spherical microphone array were about $2.4,3.3$, and $4.2 \mathrm{~m} / \mathrm{s}$, respectively. The spherical harmonic coefficients at frequencies 150 and $500 \mathrm{~Hz}$ are shown in Fig. 6 for the wind speed of $4.2 \mathrm{~m} / \mathrm{s}$. It seems that the spherical harmonic coefficients for the wind noise do not have a clear pattern and the energy is irregularly distributed along the mode number in the spherical harmonic domain, which is different from that for the sound signals shown in Fig. 4. To quantify the difference, Table II summarizes the cumulative energy ratio for the wind noise at different wind speeds.

It can be seen that less than $1 \%$ wind noise energy is contained in the first mode at $150 \mathrm{~Hz}$, while less than $10 \%$ wind noise energy is contained in the first four modes at $500 \mathrm{~Hz}$. This is dramatically different from the sound signals in Table I, where over $90 \%$ of sound energy is contained in the first mode at $150 \mathrm{~Hz}$, and in first four modes at $500 \mathrm{~Hz}$. In the acoustic measurements, if less than $1 \%$ and $10 \%$ wind noise energy are retained, the WNRs are over 20 and $10 \mathrm{~dB}$, respectively. Therefore, in comparison with Tables I and II, more than 20 and $10 \mathrm{~dB}$ WNR are expected at 150 and $500 \mathrm{~Hz}$, respectively, with a measurement accuracy of the desired SPL within $0.5 \mathrm{~dB}$.

Figure 7(a) presents the magnitude of the spherical harmonic coefficients of the wind noise at different modes and frequencies below $1000 \mathrm{~Hz}$. This shows that the wind noise is distributed irregularly in the spherical harmonic domain at
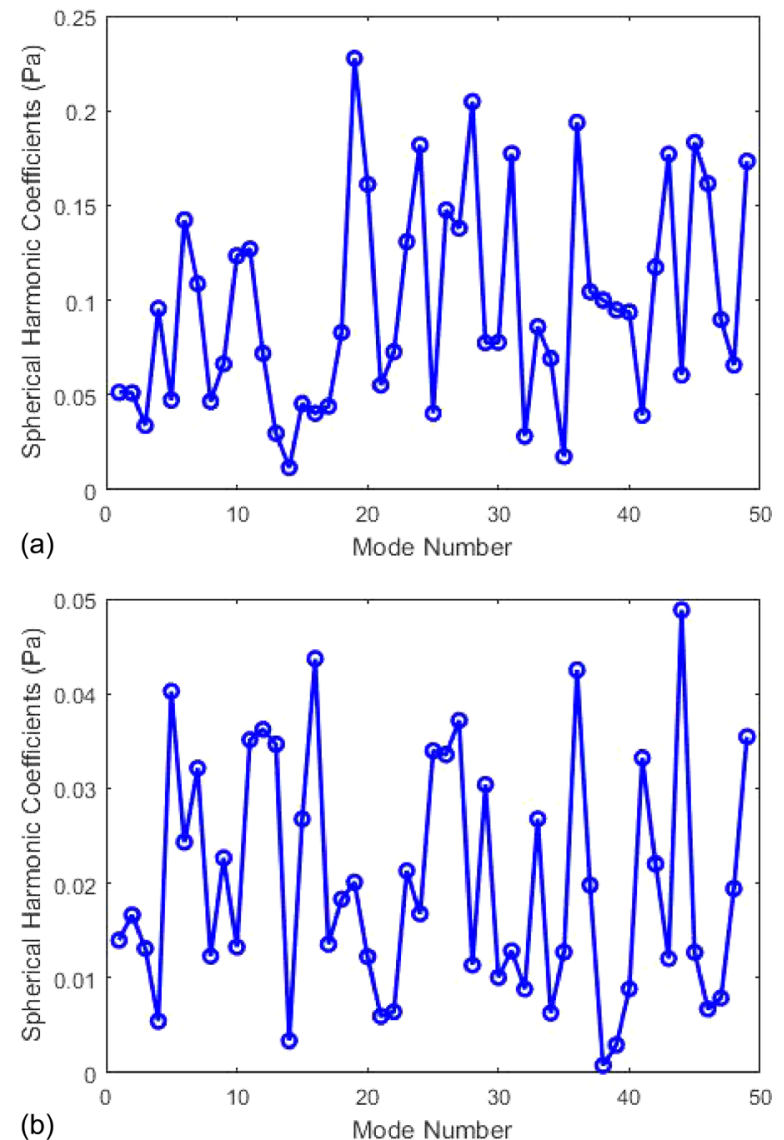

FIG. 6. (Color online) The spherical harmonic coefficients for the wind noise at the frequency of (a) $150 \mathrm{~Hz}$ and (b) $500 \mathrm{~Hz}$.

each frequency from 10 to $1000 \mathrm{~Hz}$. This implies that if the sound signal dominates in the lower order modes, then the wind noise can be reduced using the proposed low pass filter approach in the spherical harmonic domain by filtering out the higher modes. The filtered results with different threshold mode numbers are compared with the original signal in Fig. 7(b), which shows that the wind noise is reduced in the whole frequency range from 10 to $1000 \mathrm{~Hz}$ by the proposed low pass filtering in the spherical harmonic domain.

This can be observed more clearly from Fig. 8(a) for the WNR as a function of frequency. In addition, more wind noise is attenuated when fewer modes are used in the low pass filtering, as indicated in Fig. 8(b), where the overall WNR in the whole frequency range $10-1000 \mathrm{~Hz}$ is shown as a function of the threshold mode number. The overall WNR decreases from 19.0 to $3.5 \mathrm{~dB}$ as the threshold mode number

TABLE II. The cumulative energy ratio (\%) for the wind noise at different speeds.

\begin{tabular}{cccccccc}
\hline \hline Modes $\left(L_{0}\right)$ & & 1 & 2 & 3 & 4 & 5 & 10 \\
\hline$U=2.4 \mathrm{~m} / \mathrm{s}$ & $150 \mathrm{~Hz}$ & 0.004 & 0.007 & 0.008 & 0.025 & 0.026 & 0.131 \\
& $500 \mathrm{~Hz}$ & 0.012 & 0.021 & 0.023 & 0.033 & 0.063 & 0.162 \\
$U=3.3 \mathrm{~m} / \mathrm{s}$ & $150 \mathrm{~Hz}$ & 0.007 & 0.010 & 0.030 & 0.046 & 0.059 & 0.117 \\
& $500 \mathrm{~Hz}$ & 0.001 & 0.016 & 0.036 & 0.051 & 0.092 & 0.138 \\
$U=4.2 \mathrm{~m} / \mathrm{s}$ & $150 \mathrm{~Hz}$ & 0.004 & 0.074 & 0.081 & 0.092 & 0.129 & 0.148 \\
& $500 \mathrm{~Hz}$ & 0.006 & 0.010 & 0.015 & 0.031 & 0.034 & 0.104 \\
\hline \hline
\end{tabular}



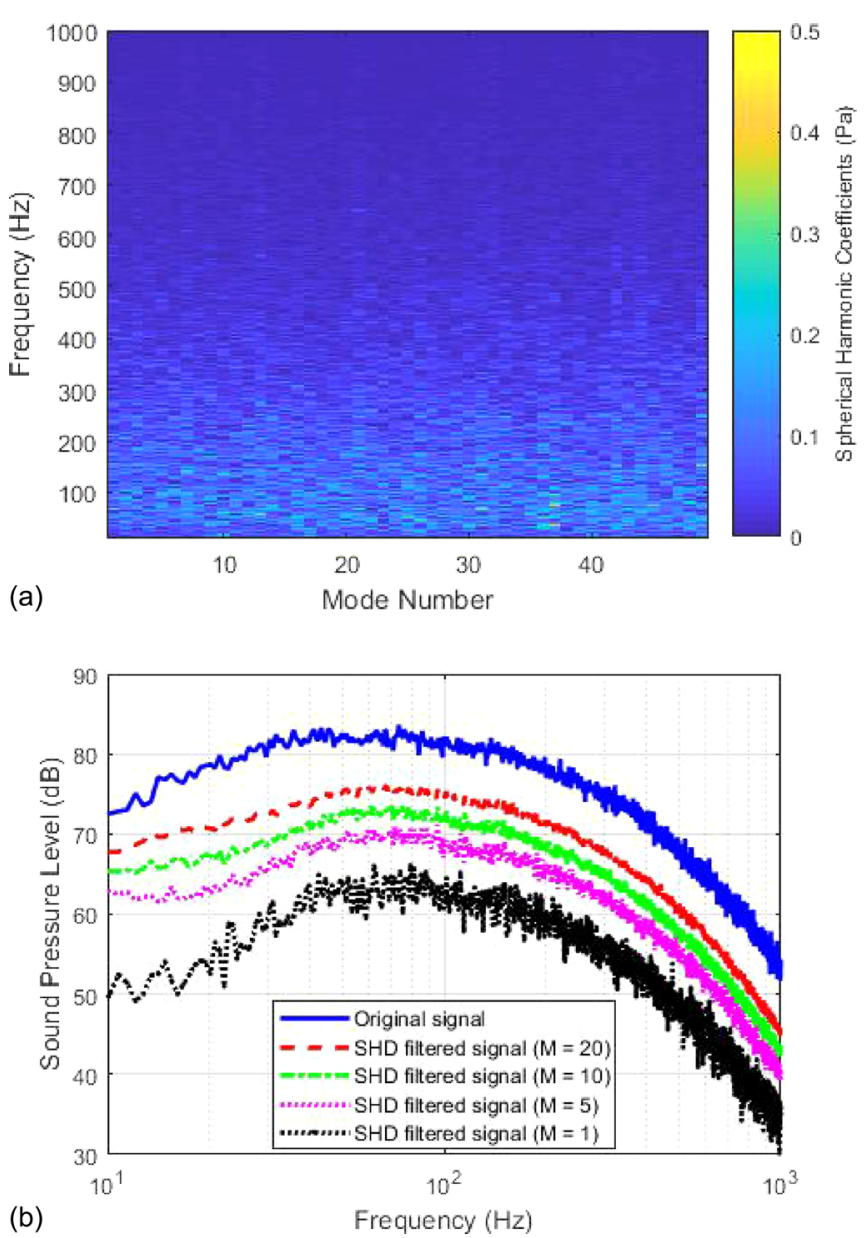

FIG. 7. (Color online) (a) The magnitude of the spherical harmonic coefficients of the wind noise at different mode and different frequency, and (b) the comparison of the original signal and the spherical harmonic domain filtered signal with different threshold mode number.

increases from one to 48 . It is noteworthy that maintaining the first spherical harmonic coefficient in the proposed method is equivalent to spatial averaging along the sphere (Rafaely, 2015). The spatial averaging provides the largest WNR, which is obtained by using the proposed low pass filter method in the spherical harmonic domain with $M=1$. It can be used as a reference level to be compared to the results with other spherical harmonic orders.

The above results demonstrate that the spherical harmonic coefficients for the wind noise are irregularly distributed at each frequency in the spherical harmonic domain, as opposed to the sound signal where the sound energy dominates in the first few spherical harmonic modes. This difference can be utilized to reduce wind noise while retaining the desired sound signal with the proposed low pass filter method in the spherical harmonic domain.

The physical reason for the difference between sound signal and wind noise is due to different generation mechanism between them. The sound signal propagates as an acoustic wave, which has the diffraction property, e.g., the sound wave can propagate beyond obstacles (the spherical microphone array here) when the wavelength is larger than the obstacle. This explains that the sound pressure at the front and rear of the sphere are similar, as shown in
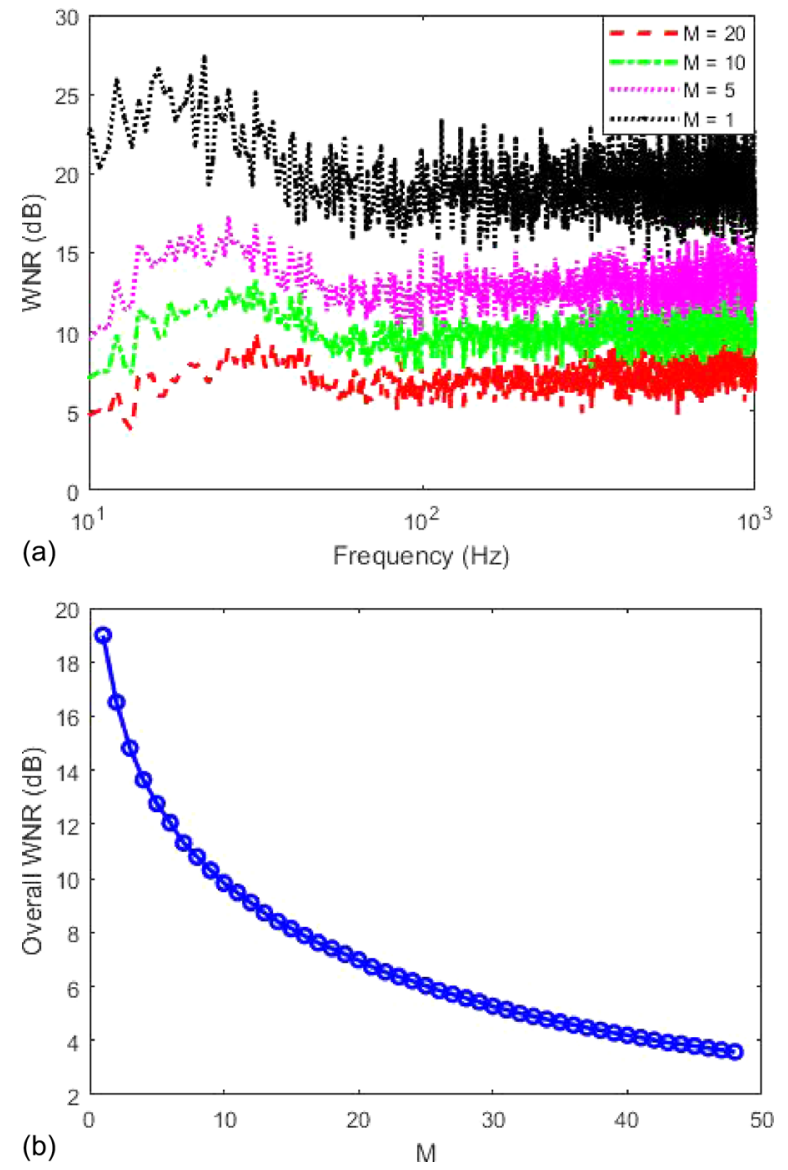

FIG. 8. (Color online) (a) WNR (dB) as a function of frequency for various threshold mode number $M$, and (b) overall WNR (dB) as a function of the threshold mode number $M$.

Fig. 3(a). However, the wind noise is caused by turbulent fluctuations, which is convected by the mean air flow. Different from wave propagation, the flow convection cannot diffract beyond obstacles, so the wind noise at the front is much larger than that at the rear of the sphere, as shown in Fig. 3(b). The different physical mechanisms of sound wave and wind noise cause the different characteristics in the spherical harmonic domain. In addition, the mean wind speed is much lower than the speed of sound, hence the turbulence wavelength is much smaller than the acoustic wavelength. This may also contribute to the difference between sound signal and wind noise in the spherical harmonic domain, which will be studied in detail in the future.

\section{Wind noise reduction (WNR) in the spherical harmonic domain}

To investigate the performance of the proposed spherical harmonic domain low pass filter method when retrieving at sound signal from wind noise, a noisy sound signal was recorded with the spherical microphone array with both the loudspeaker and fan operating. A single-tonal sound signal at different frequencies was played through the loudspeaker to conduct two sets of experiments.

In the first set of experiments, the SPL of the sound signal was higher than the wind noise and the results are shown in Fig. 9, where the spherical harmonic coefficients 

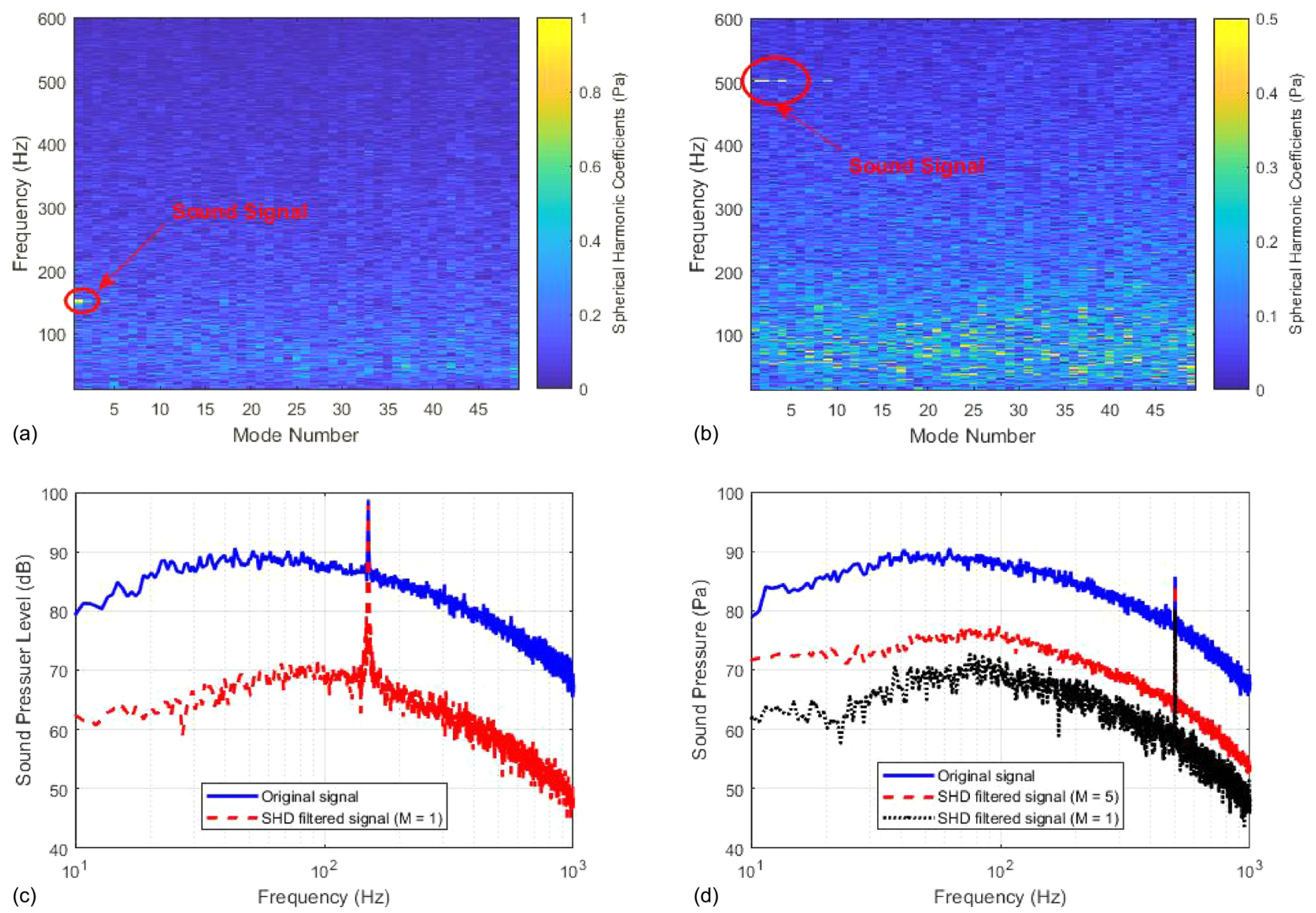

FIG. 9. (Color online) The magnitude of the spherical harmonic coefficients for the wind noise contaminated sound signal at different modes frequencies for a sound signal at (a) $150 \mathrm{~Hz}$ and (b) $500 \mathrm{~Hz}$, and comparison of the spherical harmonic domain low pass filtered signal with the original signal for the sound signal at (c) $150 \mathrm{~Hz}$ and (d) $500 \mathrm{~Hz}$. The sound signal at 150 and $500 \mathrm{~Hz}$ are 10 and $8 \mathrm{~dB}$ higher than the wind noise, respectively.

are presented for the noisy sound signal when the frequency of the sound signal is 150 and $500 \mathrm{~Hz}$, respectively. In Fig. 9, the SPL of the desired sound signal is 10 and $8 \mathrm{~dB}$ higher than the wind noise at 150 and $500 \mathrm{~Hz}$, respectively. In this case, the difference between the sound and the wind noise in the spherical harmonic domain can be observed because the sound energy is higher than the wind noise at the same frequency, as illustrated by the red marker ellipses in Figs. 9(a) and 9(b). On the other hand, the wind noise energy is irregularly distributed across the frequency range in the spherical harmonic domain, which is consistent with Fig. 7(a).

These observations indicate that the wind noise can be reduced by the proposed low pass filter method in the spherical harmonic domain as discussed in Sec. III C. The low pass filtered results are compared with the original signal in Figs. 9(c) and 9(d) for the single-tonal sound of frequency 150 and $500 \mathrm{~Hz}$, respectively. It can be observed from Fig. 9(c) that when only the first mode is kept in the spherical harmonic domain, the wind noise is reduced by $19.2 \mathrm{~dB}$ across the whole frequency range from 10 to $1000 \mathrm{~Hz}$ and the desired SPL at $150 \mathrm{~Hz}$ is retained with an error of $0.5 \mathrm{~dB}$. For the $500 \mathrm{~Hz}$ tonal sound in Fig. 9(d), with only the first mode is retained in the spherical harmonic domain $(M=1)$, the wind noise is significantly reduced by
19.2 $\mathrm{dB}$ but the desired sound signal is also degraded by $5.5 \mathrm{~dB}$. When the first five modes are retained in the spherical harmonic domain $(M=5)$, the wind noise is reduced by $13.0 \mathrm{~dB}$ across the whole frequency range from 10 to $1000 \mathrm{~Hz}$, and the desired SPL is degraded by $1.0 \mathrm{~dB}$.

The above results demonstrate that the proposed low pass filter method in the spherical harmonic domain can reduce the measured wind noise by $13.0 \mathrm{~dB}$ without degrading the sound signal when the SPL of the sound signal is higher than the wind noise. In practical applications, the SPL of the sound signal might be lower than the wind noise. In this situation, the difference between the sound signal and wind noise cannot be seen in the spherical harmonic domain, as illustrated by Figs. 10(a) and 10(b). However, the spherical harmonic domain low pass filtered results in Figs. 10(c) and 10(d) demonstrate that the proposed method can still extract the desired sound signal from wind noise. For the $150 \mathrm{~Hz}$ tonal sound signal with a SPL of $84 \mathrm{~dB}(3 \mathrm{~dB}$ lower than the wind noise), the low pass filtered results with $M=1$ and $M=5$ obtain the same SPL of $84 \mathrm{~dB}$ at $150 \mathrm{~Hz}$ in Fig. 9(c). For the $500 \mathrm{~Hz}$ tonal sound signal with a SPL of $71 \mathrm{~dB}(8 \mathrm{~dB}$ lower than the wind noise), the low pass filtered results with $M=5$ and $M=10$ derive almost the same SPL of $70 \mathrm{~dB}$ at $500 \mathrm{~Hz}$, which are both about $5 \mathrm{~dB}$ higher than that with $M=1$ as shown in Fig. 9(d). 

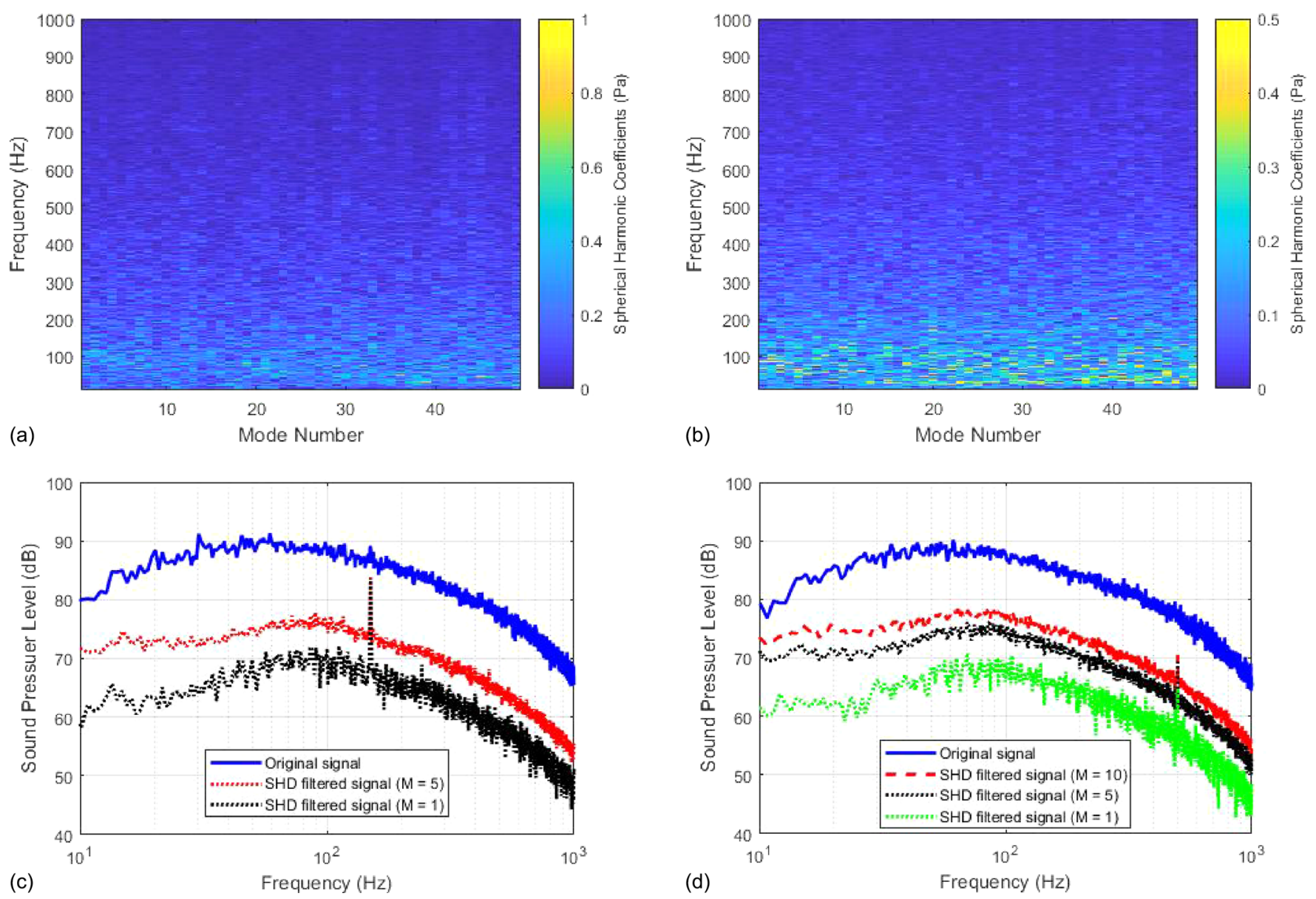

FIG. 10. (Color online) The magnitude of the spherical harmonic coefficients of the wind noise contaminated sound signal at different mode and different frequency for sound signal at (a) $150 \mathrm{~Hz}$ and (b) $500 \mathrm{~Hz}$, and comparison of the spherical harmonic domain low pass filtered signal with the original signal for the sound signal at (c) $150 \mathrm{~Hz}$ and (d) $500 \mathrm{~Hz}$. The SPL of the sound signal at 150 and $500 \mathrm{~Hz}$ are 3 and $8 \mathrm{~dB}$ lower than the wind noise, respectively.

To further evaluate the performance of the proposed method, a multi-tonal sound signal consisting of 125,250 , and $500 \mathrm{~Hz}$ sounds was recorded in the presence of wind noise. The SPL of the $125 \mathrm{~Hz}$ sound was 94 and $7 \mathrm{~dB}$ higher than the wind noise, the SPL of the $250 \mathrm{~Hz}$ sound was $82 \mathrm{~dB}$ which is the same level as the wind noise, and the SPL of the $500 \mathrm{~Hz}$ sound was 72 and $2.5 \mathrm{~dB}$ lower than the wind noise. The spherical harmonic domain low pass filtered signals with different threshold mode numbers are compared with the original signal in Fig. 11. It can be seen that when only the first mode is retained $(M=1)$, the error for 250 and $500 \mathrm{~Hz}$ sounds is 1.5 and $3.0 \mathrm{~dB}$, respectively, although the error for the $125 \mathrm{~Hz}$ sound is within $0.5 \mathrm{~dB}$. When the first five modes are retained $(M=5)$, the error is within $1.0 \mathrm{~dB}$ for all the three tonal signals, and the wind noise is reduced by $10 \mathrm{~dB}$. With more than five modes retained (e.g., $M=10$ ), there is almost no further improvement in the measurement accuracy of the desired sound signals, but the WNR is decreased by $3 \mathrm{~dB}$. Therefore, the threshold mode number $M=5$ is considered appropriate for this case, with a $10 \mathrm{~dB}$ WNR and measurement error within $1.0 \mathrm{~dB}$.

In summary, the above results demonstrate that the proposed low pass filter method in the spherical harmonic domain can extract the desired sound signal from the noisy signal even when the SPL of the sound signal is lower than the wind noise. In theory, more than 20 and $10 \mathrm{~dB}$ measured
WNR are expected at 150 and $500 \mathrm{~Hz}$, respectively, with a measurement accuracy of the desired SPL within $0.5 \mathrm{~dB}$, as shown in Tables I and II. In the experiments, 19.2 and $13.2 \mathrm{~dB}$ WNRs were observed at 150 and $500 \mathrm{~Hz}$, respectively, with the measurement accuracy of the desired SPL within $1.0 \mathrm{~dB}$. Experimental results with the multi-tonal sound signals show a WNR of $10 \mathrm{~dB}$ with the measurement error within $1.0 \mathrm{~dB}$ for all three tonal sounds.

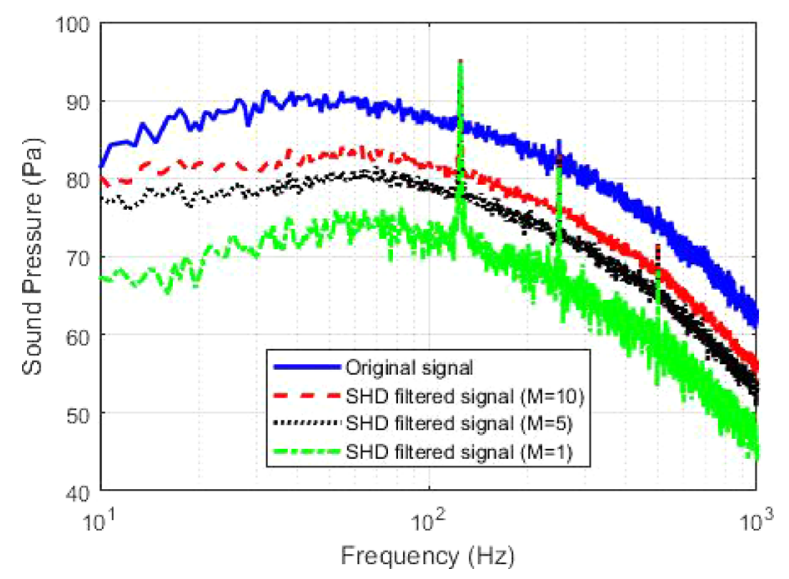

FIG. 11. (Color online) Comparison of the spherical harmonic domain low pass filtered signal with the original signal for the multi-tonal sound signal consisting of 125,250 , and $500 \mathrm{~Hz}$ tonal sound. 
The feasibility of the proposed method for outdoor WNR was verified by an outdoor experiment, which is not shown here for the sake of brevity. Although the turbulence length scale of outdoor wind is much larger than that of the wind from the fan in this paper, the results obtained by the proposed method are similar. The physical reason is that the proposed method utilized the different properties of sound signals and wind noise. Wind noise is the turbulent fluctuations convected by the mean air flow, rather than propagating acoustic wave, therefore it cannot diffract to the rear of the sphere. Therefore the property of wind noise and sound signal is much different in the spherical harmonic domain, which is utilized by the proposed method for WNR without degradation of sound signals.

\section{CONCLUSIONS}

This paper utilized a portable rigid spherical microphone array to mitigate wind noise without degrading the SPL of the desired sound signal to be measured. The wind noise was found to be irregularly distributed in the spherical harmonic domain at each frequency, contrasting with the sound signal that dominates in the first few spherical harmonic modes. A low pass filter method in the spherical harmonic domain was proposed to reduce wind noise while preserving the SPL of the desired sound signal. Experimental results showed a 19.2 and $13.2 \mathrm{~dB}$ WNR at 150 and $500 \mathrm{~Hz}$, respectively, with the measurement error of the desired sound signal within $1.0 \mathrm{~dB}$, even when the SPL of the sound signal is $8 \mathrm{~dB}$ lower than the wind noise. The proposed method was also validated with the measurements of the multi-tonal sound signals in the presence of wind noise, which showed a $10 \mathrm{~dB}$ WNR with the measurement error within $1.0 \mathrm{~dB}$. Future work will extend the proposed method to sound source localization using beamforming algorithms in the spherical harmonic domain.

\section{ACKNOWLEDGMENTS}

Professor John Davy and Dr. Christopher Preston of CSIRO are acknowledged for their support in the experiments. This research was supported under the Australian Research Council's Linkage Project funding scheme (LP140100740).

Abbott, J., Raspet, R., and Webster, J. (2015). "Wind fence enclosures for infrasonic wind noise reduction," J. Acoust. Soc. Am. 137, 1265-1273.

Chung, J. Y. (1977). "Rejection of flow noise using a coherence function method," J. Acoust. Soc. Am. 62, 388-395.
Collier, S. L., Raspet, R., Noble, J. M., Alberts, W. C. K., and Webster, J. (2014). "Analysis of wind noise reduction by semi-porous fabric domes," J. Acoust. Soc. Am. 136, 2139-2139.

Dauchez, N., Hayot, M., and Denis, S. (2016). "Effectiveness of nonporous windscreens for infrasonic measurements," J. Acoust. Soc. Am. 139, 3177-3181.

DeWolf, S., Walker, K. T., Zumberge, M. A., and Denis, S. (2013). "Efficacy of spatial averaging of infrasonic pressure in varying wind speeds," J. Acoust. Soc. Am. 133, 3739-3750.

Hedlin, M. A. H., and Raspet, R. (2003). "Infrasonic wind-noise reduction by barriers and spatial filters," J. Acoust. Soc. Am. 114, 1379-1386.

McGuinn, R. S., Lauchle, G. C., and Swanson, D. C. (1997). "Low flownoise microphone for active noise control applications," AIAA J. 35, 29-34.

Morgan, S., and Raspet, R. (1992). "Investigation of the mechanisms of low-frequency wind noise generation outdoors," J. Acoust. Soc. Am. 92, 1180-1183.

Rafaely, B. (2015). Fundamentals of Spherical Array Processing (Springer, Berlin), Vol. 1, 199 pp., available at https://link.springer.com/book/ 10.1007\%2F978-3-662-45664-4.

Raspet, R., Webster, J., and Dillion, K. (2006). "Framework for wind noise studies," J. Acoust. Soc. Am. 119, 834-843.

Shams, Q. A., Zuckerwar, A. J., and Sealey, B. S. (2005). "Compact nonporous windscreen for infrasonic measurements," J. Acoust. Soc. Am. 118, 1335-1340.

Shepherd, I. C., and La Fontaine, R. F. (1986). "Microphone screens for acoustic measurement in turbulent flows," J. Sound Vib. 111, 153-165.

Shields, F. D. (2005). "Low-frequency wind noise correlation in microphone arrays," J. Acoust. Soc. Am. 117, 3489-3496.

Shust, M. (1998). "Active removal of wind noise from outdoor microphone using local velocity measurements," Michigan Technological University, 133 pp.

Shust, M. R., and Rogers, J. C. (1998). "Active removal of wind noise from outdoor microphones using local velocity measurements," J. Acoust. Soc. Am. 104, 1781.

Strasberg, M. (1988). "Dimensional analysis of windscreen noise," J. Acoust. Soc. Am. 83, 544-548.

van den Berg, G. P. (2006). "Wind-induced noise in a screened microphone," J. Acoust. Soc. Am. 119, 824-833.

VisiSonics (2015). VisiSonics 5/64 Audio Visual Camera, Available: http:// visisonics.com/download/audiovisual-camera-pamphlet/ (Last viewed 21 February 18).

Wilson, D., Greenfield, R., and White, M. (2007). "Spatial structure of lowfrequency wind noise," J. Acoust. Soc. Am. 122, EL223-EL228.

Wilson, D. K., and White, M. J. (2010). "Discrimination of wind noise and sound waves by their contrasting spatial and temporal properties," Acta Acust. Acust. 96, 991-1002.

Zhao, S., Cheng, E., Qiu, X., Burnett, I., and Liu, J. C. (2016). "Pressure spectra in turbulent flows in the inertial and the dissipation ranges," J. Acoust. Soc. Am. 140, 4178-4182.

Zhao, S., Cheng, E., Qiu, X., Burnett, I., and Liu, J. C. (2017a). "Wind noise spectra in small Reynolds number turbulent flows," J. Acoust. Soc. Am. 142, 3227-3233.

Zhao, S., Cheng, E., Qiu, X., Burnett, I., and Liu, J. C. (2018). "Spatial decorrelation of wind noise with porous microphone windscreens," J. Acoust. Soc. Am. 143, 330-339.

Zhao, S., Dabin, M., Cheng, E., Qiu, X., Burnett, I., and Liu, J. C. (2017b). "On the wind noise reduction mechanism of porous microphone windscreens," J. Acoust. Soc. Am. 142, 2454-2463. 